\title{
Efficacy of manual therapy interventions in management of lumbar prolapsed intervertebral disc: A pilot randomized controlled trial
}

\author{
Varun Singh, Manoj Malik \\ Department of Physiotherapy, Guru Jambheshwar University of Science and Technology, Hisar, India
}

\begin{abstract}
Background. This pilot trial reports the initial estimates of the efficacy of manual therapy interventions in lumbar prolapsed intervertebral disc and determines the feasibility and acceptability of full powered "randomized controlled trial" on efficacy of "spinal mobilization with leg movement (SMWLM)", high velocity low amplitude thrust (HVLA) and neural mobilization (NM) in lumbar PIVD (Prolapsed Inter-Vertebral Disc) and pilot data will be used to perform sample size calculation for full trial.

Material and methods. 48 subjects diagnosed lumbar PIVD were randomly distributed into 4 groups. The primary outcomes were feasibility, assessment procedure, retention rate, adherence and acceptability to the intervention. The secondary outcomes measures were pain, disability and straight leg raise (SLR) range of motion.

Results. 90 subjects were screened based on selection criteria. Out of them, 50 (55.55\%) were eligible. 48(96\%) subjects accepted to participate in study. Baseline data of all the groups was similar but post-intervention score were significant when compared the data between the groups. Highest mean change for visual analog scale (VAS), oswestry disability index (ODI) and SLR were found in SMWLM group. No adverse effects were reported by subjects. Results also suggest that the outcome measures were feasible and acceptable and the treatment considered as the beneficial approach.

Conclusions. Present study suggests that it is feasible and acceptable to do a fully powered "randomized controlled trial (RCT)" to evaluate the efficacy of manual therapy interventions in management of lumbar PIVD. This study also reveals that manual therapy interventions are effective in management of lumbar PIVD.
\end{abstract}

Keywords: physiotherapy, manual therapy, lumbar PIVD, neural mobilization, manipulation

\section{INTRODUCTION}

Low back pain is identified as a major health issue across the globe and getting worse as reported by "Global Burden of Disease Survey" in 2016 [1]. It is most common cause for patients to visit a health professional and also most common symptom associated with lumbar disc herniation/prolapse $[2,3]$. In general population, $60 \%-80 \%$ prevalence is due to disc herniation [3]. Approximately $10 \%$ cases have symptoms of sciatica, with lifetime prevalence from $13 \%$ to $40 \%$ [4]. Sciatica associated with low back pain leads to delayed recovery, persistent disa- bility and economical burden [4]. A variety of interventions are used for management of lumbar disc herniation with varying degree of success. Recent studies show beneficial effects of manual therapy in lumbar disc herniation [5-8]. In manual therapy hands-on techniques are delivered for assessment, diagnosis, management and prognosis [5-8]. However, for lumbar disc herniation, there are no standardized treatments protocols for interventions, more treatment options are need of time [9]. Therefore, the primary aim was to determine the feasibility, retention rate, adherence rate and acceptability 
of full powered randomized controlled trial. The secondary aim was to rule out the initial estimates of the efficacy of manual therapy interventions in lumbar prolapsed intervertebral disc.

\section{MATERIAL AND METHODS}

This pilot study was done in accordance with the Consolidated Standards of Reporting Trials (CONSORT) guidelines 2010 for randomized pilot or feasibility trials.

\section{Study design and setting}

Study design of pilot randomized controlled trial was approved by the Institutional Ethical Committee (IEC) of University. The trial was registered with the Clinical Trial Registry India (trial number CTRI/2020/01/023037).

Subjects with diagnosed lumbar disc herniation/ prolapse having radiological evidence in MRI (magnetic resonance imaging). Subjects with age between 18-50 years, having unilateral radiculopathy pain below knee.

Exclusion criteria: Sequestrated disc, osteoporosis, pregnancy, tuberculosis, recent injuries like fractures, grade 3 ligament injuries, previous spinal surgery, conditions like spondylolisthesis, tumor in lumbar spine and hyper mobility.

Total 48 subjects were taken, 12 subjects in each group as a thumb rule for minimum sample size in pilot study [10,11].

Computer generated random number table was used to allocate the participants in experimental and control groups.

\section{Outcome measures}

The primary outcomes were feasibility, retention rate, adherence rate and acceptability. The secondary outcomes were pain, disability and SLR ROM. Pain was measured from $0 \mathrm{~cm}$, "no pain," to $10 \mathrm{~cm}$, "the worst pain ever" on visual analog scale. Visual analog scale has a high inter observer reliability in musculoskeletal conditions [12]. For disability evaluation in low back pain patients, ODI is useful and is used most commonly [13-15]. ODI contains 10 items. Higher score indicates greater disability and vice-versa.

\section{Study procedures}

Pain, disability and SLR range of motion were assessed using VAS, ODI and digital goniometer, respectively. Outcomes were assessed every week after intervention. In group A, SMWLM, lumbar traction (for 15 minutes) and interferential therapy (for 15 minutes) was given. Treatment protocol was given for twelve sessions carried out over four con- secutive weeks. In group B, HVLA thrust, lumbar traction (for 15 minutes) and interferential therapy (for 15 minutes) was given for twelve sessions over a period of four consecutive weeks. In group C, neural mobilization, lumbar traction (for 15 minutes) and interferential therapy (for 15 minutes) was given for twelve sessions carried out over four consecutive weeks. In group D, lumbar traction (for 15 minutes) and interferential therapy (for $15 \mathrm{~min}$ utes) was given for twelve sessions carried out over four consecutive weeks.

\section{SMWLM Group}

Patient was side lying with painful leg up. Therapist bends over and places thumb on side of spinous process of upper vertebra at site of lesion. A firm transverse pressure was sustained while assistant was instructed to perform the offending movement within pain free zone. In first treatment session, a set of three repetitions was delivered, followed by three sets of six repetitions with a thirty second rest interval between sets on consequent treatment sessions. Passive pain free overpressure to SLR was being given as progression. Along with SMWLM, intermittent lumbar traction (15 minutes) with traction force $30 \%$ of body weight and interferential therapy (15 minutes) 4 electrode crossed pattern were applied [16,17].

\section{HVLA group}

Patient was in side lying position. Therapist turned the upper trunk of patient so that rotation and lateral flexion of lumbar region takes place. Then, counter rotate in such a way so that the desired segment can be manipulated by applying high velocity low amplitude thrust within anatomical barrier. Along with HVLA, intermittent lumbar traction (15 minutes) with traction force $30 \%$ of body weight, interferential therapy (15 minutes) 4 electrode crossed pattern were applied.

\section{Neural mobilization group}

Neural tissue mobilization was delivered in stepwise manner. Step 1- Sliders; by using unaffected joint and affected joint is placed in neutral position. Step 2- Sliders; by using unaffected joint and affected joint is placed in available range of motion. Step 3- Sliders; by moving affected and unaffected joint but with or without minimum symptoms. Neural mobilization was applied for 30 seconds $\times 5$ sets. Along with neural mobilization, intermittent lumbar traction (15 minutes) with traction force $30 \%$ of body weight and interferential therapy (15 minutes) 4 electrode crossed pattern were applied. 


\section{Data analysis}

Data were analyzed using “SPSS 21.0”. Normality of data was checked by using Kolmogorov-Smirnov Test. Treatment effects was analysed by Post hoclsd. Comparisons between all groups were made by using one way ANOVA. Pre-post comparisons have done using related t-test .

\section{RESULTS}

90 subjects were screened based on selection criteria. Out of them, 50 (55.55\%) were eligible. 48(96\%) subjects accepted to participate in study (Figure 1). Baseline data of all the groups was similar (Table 1). Feasibility of outcome measure; all participants gave base line data and post-intervention data. Adherence rate; 46 out of 48 participants (95.83\%) completed all 12 sessions. Attrition rate of $4.17 \%$ was documented at the end of study. Retention rate; 46 participants (95.83\%) completed all outcome measures.

After 4 week intervention when compared within groups, significant improvement was seen. Highest mean change in VAS was shown by SMWLM group $(\mathrm{MD}=6.25 \pm 1.05 ; 95 \%$ CI 5.58 to $6.92 ; \mathrm{p}<.01)$ followed by CT group ( $\mathrm{MD}=4.09 \pm .54 ; 95 \% \mathrm{CI} 3.73$ to $4.45 ; \mathrm{p}<.01$ ) after 4 week of intervention. Highest mean change in ODI was shown by SMWLM group $(\mathrm{MD}=14.67 \pm 2.42 ; 95 \%$ CI 13.13 to 16.21 ; $\mathrm{p}$ <.01) followed by HVLA group $(\mathrm{MD}=11.91 \pm 1.51 ; 95 \% \mathrm{CI}$ 10.89 to $12.93 ; \mathrm{p}<.01$ ) after 4 week of intervention. Highest mean change in SLR was shown by SMWLM group (MD = 19.19 \pm 1.02 ; 95\%CI -19.84 to -18.55 ; $\mathrm{p}<$ .01 ) followed by HVLA group (MD = 7.64 \pm 1.88 ; $95 \% \mathrm{CI}$ -8.90 to $-6.36 ; \mathrm{p}<.01$ ) after 4 week of intervention (Table 2).

Significant difference were found in mean change in VAS score after 4 week of intervention in SMWLM group $(\mathrm{MD}=2.34 ; 95 \% \mathrm{CI} 1.69$ to 2.99 ; $\mathrm{p}<$ $.01),(\mathrm{MD}=2.83 ; 95 \% \mathrm{CI} 2.20$ to $3.47 ; \mathrm{p}<.01),(\mathrm{MD}=$ 2.16; $95 \%$ CI 1.51 to $2.81 ; \mathrm{p}<.01$ ), when compared with HVLA group, NM group and CT group respectively. There were non-significant difference in mean change in VAS in HVLA group when compared with NM group and CT group. Significant difference was found in mean change in VAS in NM group (MD $=-0.67 ; 95 \% \mathrm{CI}-1.32$ to $-0.02 ; \mathrm{p}<.01$ ) when compared with CT group. Significant difference were found in mean change in ODI score after 4 week of intervention in SMWLM group $(\mathrm{MD}=2.76$;

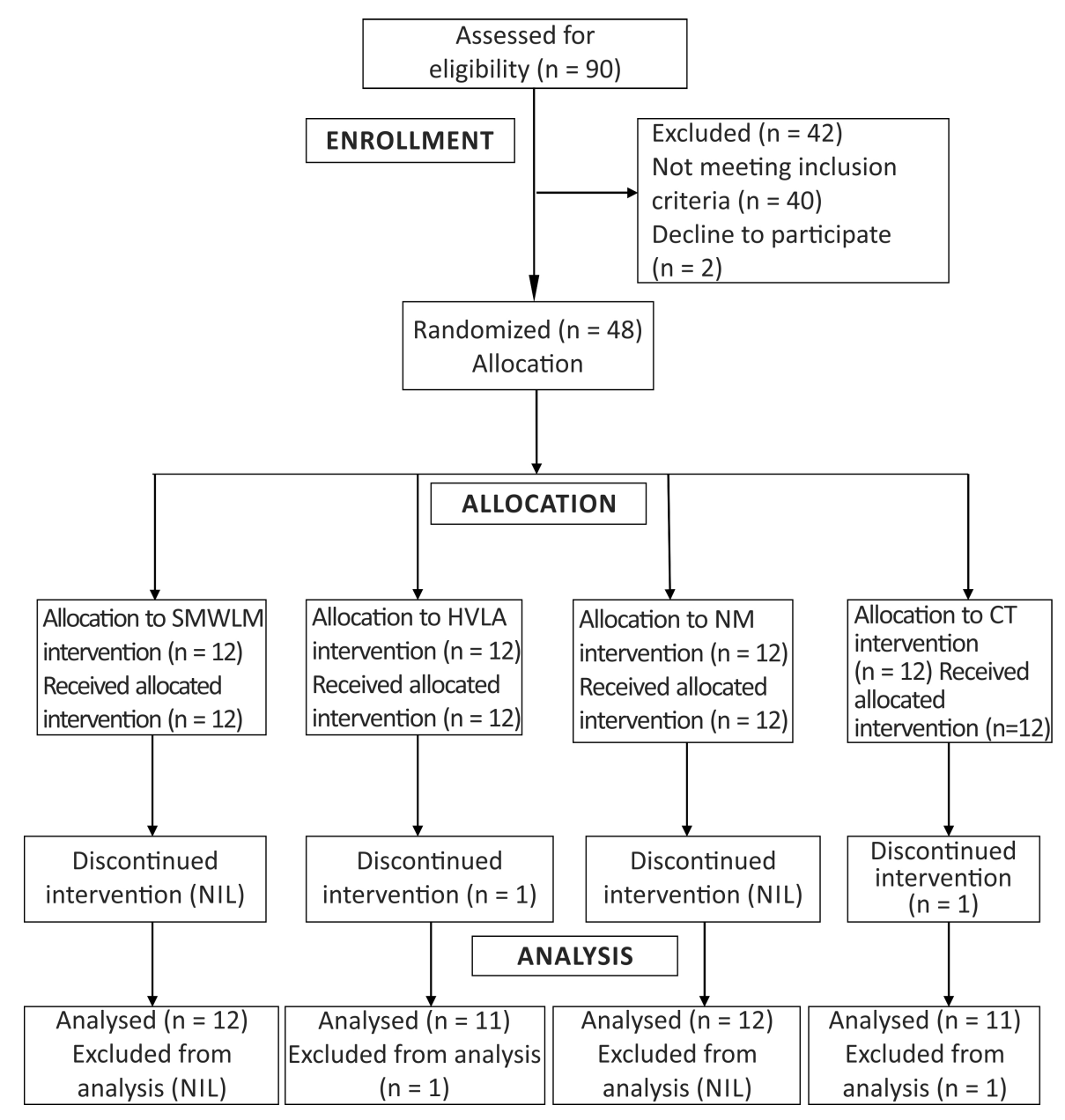

FIGURE 1. CONSORT Flow Diagram 
TABLE 1. Comparison of mean difference of VAS, ODI and SLR within different intervention groups (using paired t-test)

\begin{tabular}{|c|c|c|c|c|c|c|}
\hline & Group & Pre-intervention & Post-intervention & MD & T-test & P-value \\
\hline \multirow[t]{4}{*}{ VAS } & SMWLM & $8.00 \pm 1.04$ & $1.75 \pm 0.62$ & $6.25 \pm 1.06$ & 20.52 & $<0.01$ \\
\hline & HVLA & $7.91 \pm 0.94$ & $4.00 \pm 0.63$ & $3.91 \pm 0.54$ & 24.04 & $<0.01$ \\
\hline & NM & $7.50 \pm 1.3$ & $4.08 \pm 0.79$ & $3.42 \pm 0.79$ & 14.93 & $<0.01$ \\
\hline & $\mathrm{CT}$ & $8.27 \pm 0.79$ & $4.18 \pm 0.75$ & $4.09 \pm 0.54$ & 25.16 & $<0.01$ \\
\hline \multirow[t]{4}{*}{ ODI } & SMWLM & $26.08 \pm 2.64$ & $11.42 \pm 0.79$ & $14.67 \pm 2.42$ & 20.96 & $<0.01$ \\
\hline & HVLA & $27.73 \pm 1.79$ & $15.82 \pm 0.87$ & $11.91 \pm 1.51$ & 26.09 & $<0.01$ \\
\hline & NM & $27.42 \pm 1.73$ & $16.08 \pm 1.08$ & $11.33 \pm 1.15$ & 34.00 & $<0.01$ \\
\hline & CT & $27.82 \pm 1.33$ & $16.82 \pm 0.98$ & $11.00 \pm 1.73$ & 21.06 & $<0.01$ \\
\hline \multirow[t]{4}{*}{ SLR } & SMWLM & $48.28 \pm 1.28$ & $67.48 \pm 1.33$ & $-19.19 \pm 1.02$ & -65.39 & $<0.01$ \\
\hline & HVLA & $48.01 \pm 1.19$ & $55.65 \pm 1.47$ & $-7.64 \pm 1.88$ & -13.49 & $<0.01$ \\
\hline & NM & $48.93 \pm 1.74$ & $54.42 \pm 1.01$ & $-5.50 \pm 2.17$ & -8.77 & $<0.01$ \\
\hline & CT & $48.61 \pm 0.81$ & $55.24 \pm 0.74$ & $-6.63 \pm 1.01$ & -21.76 & $<0.01$ \\
\hline
\end{tabular}

TABLE 2. Comparison of mean change of VAS, ODI and SLR between different intervention groups (using one way ANOVA)

\begin{tabular}{|c|c|c|c|c|c|c|}
\hline Variables & SMWLM (N=12) & HVLA (N=11) & NM (N=12) & CT (N=11) & F & P \\
\hline $\begin{array}{c}\text { Mean VAS } \\
\text { change }\end{array}$ & $6.25 \pm 1.05$ & $3.91 \pm 0.54$ & $3.42 \pm 0.79$ & $4.09 \pm 0.54$ & 31.56 & .000 \\
\hline $\begin{array}{c}\text { Mean ODI } \\
\text { change }\end{array}$ & $14.67 \pm 2.42$ & $11.91 \pm 1.51$ & $11.33 \pm 1.15$ & $11.00 \pm 1.73$ & 10.44 & .000 \\
\hline $\begin{array}{c}\text { Mean SLR } \\
\text { change }\end{array}$ & $19.19 \pm 1.02$ & $7.64 \pm 1.88$ & $5.50 \pm 2.17$ & $6.63 \pm 1.01$ & 185.75 & .000 \\
\hline
\end{tabular}

95\%CI 1.26 to $4.25 ; \mathrm{p}<.01),(\mathrm{MD}=3.33 ; 95 \% \mathrm{CI}-1.87$ to $4.80 ; \mathrm{p}<.01),(\mathrm{MD}=3.67 ; 95 \% \mathrm{CI} 2.17$ to 5.16 ; $\mathrm{p}<$ .01), when compared with HVLA group, NM group and CT group respectively. There were non-significant differences in mean change in ODI in HVLA group when compared with NM group and CT group and also in NM group when compared with CT group. Significant difference were found in mean change in SLR score after 4 week of intervention in SMWLM group $(\mathrm{MD}=11.55 ; 95 \% \mathrm{CI}-12.91$ to -10.20 ; $\mathrm{p}<.01),(\mathrm{MD}=13.69 ; 95 \% \mathrm{CI}-15.02$ to $-12.37 ; \mathrm{p}<.01)$, $(\mathrm{MD}=12.56 ; 95 \% \mathrm{CI}-13.92$ to $-11.21 ; \mathrm{p}<.01)$ when

TABLE 3. Multiple comparison of mean change of VAS, ODI and SLR between different intervention groups (using one way ANOVA)

\begin{tabular}{|c|c|c|c|c|}
\hline Variable & $\begin{array}{c}\text { Group } \\
\text { intervention }\end{array}$ & $\begin{array}{c}\text { Comparison between } \\
\text { groups }\end{array}$ & $\begin{array}{c}\text { MD } \\
\text { (mean difference) }\end{array}$ & p-value \\
\hline \multirow[t]{6}{*}{ Mean VAS Change } & \multirow[t]{3}{*}{ SMWLM (N=12) } & HVLA & $2.341^{*}$ & .000 \\
\hline & & NM & $2.833^{*}$ & .000 \\
\hline & & CT & $2.159^{*}$ & .000 \\
\hline & \multirow[t]{2}{*}{ HVLA (N=11) } & NM & 0.492 & .134 \\
\hline & & CT & -.182 & .583 \\
\hline & $\mathrm{NM}(\mathrm{N}=12)$ & CT & $-.674^{*}$ & .042 \\
\hline \multirow[t]{6}{*}{ Mean ODI Change } & \multirow[t]{3}{*}{ SMWLM $(\mathrm{N}=12)$} & HVLA & $2.758^{*}$ & .001 \\
\hline & & NM & $3.333^{*}$ & .000 \\
\hline & & CT & $3.667^{*}$ & .000 \\
\hline & \multirow[t]{2}{*}{ HVLA (N=11) } & NM & 0.576 & .441 \\
\hline & & CT & 0.909 & .236 \\
\hline & $\mathrm{NM}(\mathrm{N}=12)$ & CT & 0.333 & .655 \\
\hline \multirow[t]{6}{*}{ Mean SLR Change } & \multirow[t]{3}{*}{ SMWLM $(\mathrm{N}=12)$} & HVLA & $11.555^{*}$ & .000 \\
\hline & & NM & $13.691^{*}$ & .000 \\
\hline & & CT & $12.564^{*}$ & .000 \\
\hline & \multirow[t]{2}{*}{ HVLA $(\mathrm{N}=11)$} & NM & $2.136^{*}$ & .003 \\
\hline & & CT & 1.009 & .149 \\
\hline & $\mathrm{NM}(\mathrm{N}=12)$ & CT & 1.127 & .101 \\
\hline
\end{tabular}


compared with HVLA group, NM group and CT group respectively. There were significant differences in mean change in SLR in HVLA group (MD = 2.14; $95 \%$ CI -3.49 to $-0.78 ; \mathrm{p}<.01$ ) when compared with NM group. There were non-significant difference in mean change in SLR in HVLA group and NM group when compared with CT group (Table 3 ).

\section{DISCUSSION}

The results suggest that a future "randomized controlled trial" evaluating the efficacy of manual therapy interventions in management of lumbar prolapsed intervertebral disc is feasible. Present study have recruitment rate of $96 \%$. At this rate, a fully powered "randomized controlled trial" is feasible. The retention rate of $95.83 \%$ was achieved. $95.83 \%$ of participant completed all 12 sessions which can be attributed as a good adherence rate. One of the reasons behind this success rate is flexible treatment timing as per convenience of patient. This study also compares the efficacy of SMWLM, HVLA thrust and NM in lumbar PIVD. The study reveals that these techniques, along with intermittent lumbar traction and interferential therapy, results in decrease of pain, disability and improve SLR range of motion. Outcomes measures improved over 4 weeks of intervention from baseline.

SMWLM is effective in lumbar radiculopathy with pain beyond the knee joint [16]. It corrects the positional fault, which unloads the pressure from compressed nerve and help in pain management [17]. However, previous studies on effectiveness of SMWLM are mostly case studies, [16-19] with only a few randomized controlled trials [20-22]. These researches have limited outcome measures and time duration that creates a need for further studies.

High velocity low amplitude (HVLA) thrust is a type of manual intervention for opening of restricted range of motion and alignment correction. It is useful in lumbar disc herniation [23,24]. Pain modulation takes place at dorsal horn by altering neuroplasticity and central sensitization. Spinal mobiliza-

\section{REFERENCES}

1. Hartvigsen J, Hancock MJ, Kongsted A, et al. What low back pain is and why we need to pay attention. Lancet. 2018;391:2356-67.

2. Balagué $F$, Mannion AF, Pellisé $F$, et al. Non-specific low back pain. Lancet. 2012;379:482-91.

3. Awad JN, Moskovich R. Lumbar disc herniations: surgical versus nonsurgical treatment. Clin Orthop Relat Res. 2006;443:183-97.

4. Tubach F, Beauté J, Leclerc A. Natural history and prognostic indicators of sciatica. J Clin Epidemiol. 2004;57:174-79.

5. Clar C, Tsertsvadze A, Hundt GL, et al. Clinical effectiveness of manual therapy for the management of musculoskeletal and nonmusculoskeletal conditions: systematic review and update of UK evidence report. Chiropr Man Therap. 2014;22:1-34. tion inhibits pain by counter-irritant effect [25]. Up to $13.3 \%$ change in sympathetic nervous system activity seen after application of unilateral manual technique to lumbar spine [26]. Haas et al. recorded the lasting effects of manipulation and effects were long lasting up to 5 hours post-manipulation [27]. Studies conclude that the mechanism of action of manipulation is by modulating the excitability of dorsal horn that produces immediate effects [2830].

Neural mobilization leads to improve neural gliding and helps in restoring mobility [31]. Bertolini et al. stated that demyelination and neural edema occurs due to reduced microcirculation caused by compression of nerve root [32]. The oscillatory movements applied during neural mobilization decreases inflammation and neural tissue hypoxia. It is also believed that nerve movement within available pain free range decreases the mechanosensitivity [32]. It breaks the adhesions and improves neuro-dynamics. It relieves pain as well as improves SLR in lumbar disc herniation patients.

\section{Limitations and future studies}

Lack of large sample size and absence of long term outcomes follow up are limitations of this study. In future studies, large sample size and implementation of manual therapy in bilateral radiculopathy as well as combination of therapy may be used.

\section{CONCLUSIONS}

Present study suggests that it is feasible and acceptable to do a fully powered RCT to evaluate the efficacy of manual therapy interventions in management of lumbar prolapsed intervertebral disc. This study reveals that there was significant difference in pain, disability and SLR range of motion in all groups. Present study reveals that highest mean change for VAS, ODI and SLR were found in SMWLM group. SMWLM can be used in management of lumbar prolapsed intervertebral disc.

Conflict of interest: none declared Financial support: none declared

6. Assendelft WJ, Morton SC, Yu El, et al. Spinal manipulative therapy for low back pain: a meta-analysis of effectiveness relative to other therapies. Ann Intern Med. 2003;138:871-81.

7. Bronfort $\mathrm{G}$, Haas M, Evans RL, Bouter LM. Efficacy of spinal manipulation and mobilization for low back pain and neck pain: a systematic review and best evidence synthesis. Spine. 2004;4:335-56.

8. Bronfort $\mathrm{G}$, Haas M, Evans $\mathrm{R}$, et al. Effectiveness of manual therapies: the UK evidence report. Chiropr Osteopat. 2010;18:1-33.

9. Dan-Azumi MS, Bello B, Rufai SA, Abdulrahman MA. Surgery versus conservative management for lumbar disc herniation with radiculopathy: A systematic review and meta-analysis. J. Health Sci. 2018;8:42-53. 
10. Julious SA. Sample size of 12 per group rule of thumb for a pilot study. Pharmaceutical Statistics: J Appl Stat. 2005;4:287-91.

11. Whitehead AL, Julious SA, Cooper CL, Campbell MJ. Estimating the sample size for a pilot randomised trial to minimise the overall trial sample size for the external pilot and main trial for a continuous outcome variable. Stat Methods Med Res. 2016;25:1057-73.

12. Boonstra AM, Preuper HRS, Reneman MF, et al. Reliability and validity of the visual analogue scale for disability in patients with chronic musculoskeletal pain. Int J Rehabil Res. 2008;31:165-69.

13. Delitto A, George SZ, Van Dillen LR, et al. Low back pain. J Orthop Sports Phys Ther. 2012;42:A1-57.

14. Chapman JR, Norvell DC, Hermsmeyer JT, et al. Evaluating common outcomes for measuring treatment success for chronic low back pain. Spine. 2011;36: S54-68.

15. Fairbank JC, Pynsent PB. The Oswestry disability index. Spine. 2000;25:2940-53.

16. Mulligan BR. Spinal mobilisations with leg movement (further mobilisations with movement). J Man Manip Ther. 1995;3:25-27.

17. Mulligan BR. Update on spinal mobilisations with leg movement. J Man Manip Ther. 1997;5:184-87.

18. Wilson E. Mobilisation with movement and adverse neural tension: an exploration of possible links. Manipulative Physiotherapist. 1995;27:40-46.

19. Wilson E. Central facilitation and remote effects: treating both ends of the system. Man Ther. 1997;2:165-68.

20. Yadav S, Nijhawan MA, Panda P. Effectiveness of spinal mobilization with leg movement (SMWLM) in patients with lumbar radiculopathy (L5/S1 nerve root) in lumbar disc herniation. Int J Physiother Res. 2014;2:712-18.

21. Das SMS, Dowle P, lyengar R. Effect of spinal mobilization with leg movement as an adjunct to neural mobilization and conventional therapy in patients with lumbar radiculopathy: Randomized controlled trial. J Med Sci Res. 2018;6:11-19.

22. Ahmed N, Khan Z, Chawla C. Comparison of Mulligan's spinal mobilization with limb movement (SMWLM) and neural tissue mobilization for the treatment of lumbar disc herniation: a randomized clinical trial. J Novel Physiother. 2016;6:1-9.

23. Kirkaldy-Willis WH, Cassidy JD. Spinal manipulation in the treatment of low-back pain. Can Fam Physician. 1985;31:535-40.

24. Hondras $\mathrm{MA}$, Long $\mathrm{CR}$, $\mathrm{Cao} \mathrm{Y}$, et al. A randomized controlled trial comparing 2 types of spinal manipulation and minimal conservative medical care for adults 55 years and older with subacute or chronic low back pain. J Manipulative Physiol Ther. 2009;32:330-43.

25. Perry J, Green A, Singh S, Watson P. A preliminary investigation into the magnitude of effect of lumbar extension exercises and a segmental rotatory manipulation on sympathetic nervous system activity. Man Ther. 2011;16:190-95.

26. Perry J, Green A. An investigation into the effects of a unilaterally applied lumbar mobilisation technique on peripheral sympathetic nervous system activity in the lower limbs. Man Ther. 2008;13:492-99.

27. Haas M, Groupp E, Panzer D, et al. Efficacy of cervical endplay assessment as an indicator for spinal manipulation. Spine. 2003;28:1091-96.

28. Boal RW, Gillette RG. Central neuronal plasticity, low back pain and spinal manipulative therapy. J Manipulative Physiol Ther. 2004;27:31426.

29. Bialosky JE, Bishop MD, Robinson ME, et al. Spinal manipulative therapy has an immediate effect on thermal pain sensitivity in people with low back pain: a randomized controlled trial. Phys Ther. 2009;89:1292-03.

30. Bialosky JE, Bishop MD, Robinson ME, et al. The influence of expectation on spinal manipulation induced hypoalgesia: an experimental study in normal subjects. BMC Musculoskelet Disord. 2008;9:1-9.

31. Sarkari E, Multani NK. Efficacy of neural mobilisation in sciatica. JESP. 2007;3:136-141.

32. Bertolini GR, Silva TS, Trindade DL, et al. Neural mobilization and static stretching in an experimental sciatica model: an experimental study. Braz J Phys Ther. 2009;13:493-98. 